\title{
Implementation of quantum gates based on geometric phases accumulated in the eigenstates of periodic invariant operators
}

\author{
L. B. Shao, ${ }^{1,2}$ Z. D. Wang, ${ }^{1,2, *}$ and D. Y. Xing ${ }^{1}$ \\ ${ }^{1}$ National Laboratory of Solid State of Microstructure and Department of Physics, Nanjing University, Nanjing 210093, China \\ ${ }^{2}$ Department of Physics and Center of Theoretical and Computational Physics, The University of Hong Kong, Pokfulam Road, \\ Hong Kong, China \\ (Received 24 October 2006; published 16 January 2007)
}

\begin{abstract}
We propose a strategy to physically implement a universal set of quantum gates based on geometric phases accumulated in the nondegenerate eigenstates of a designated invariant operator in a periodic physical system. The system is driven to evolve in such a way that the dynamical phase shifts of the invariant operator eigenstates are identical (or mod $2 \pi$ ) while the corresponding geometric phases are nontrivial. We illustrate how this strategy works in a simple but typical NMR-type qubit system.
\end{abstract}

DOI: 10.1103/PhysRevA.75.014301

PACS number(s): 03.67.Lx, 03.65.Vf

Quantum computation, based on fundamental quantum mechanical principles such as superposition and entanglement, may provide a promising perspective to advance modern computational science $[1,2]$. So far, a lot of substantial efforts have been dedicated to the field of quantum computation and a number of significant progresses have been made [2-5]. Nevertheless, quantum computation is still facing great challenges before it can be put into practice. As one of them, how to suppress the random errors during gate operations has been paid much attention for the past years.

Recently, geometric quantum computation (GQC), expected as an intrinsical fault-tolerant scheme, was proposed by using NMR [6,7], superconducting nanocircuits $[8,9]$, trapped ions [10], or semiconducting nanostructures [11]. As is well known [12-14], for an adiabatic (or nonadiabatic) cyclic evolution, the associated total phase shift consists of both dynamic and geometric components, where the geometric phase is interpreted as a holonomy of the Hermitian fiber bundle over the parameter (projective Hilbert) space. Since the geometric phase depends only on the global geometry of the path executed in the evolution, quantum logical gates related only to pure geometric phase shifts in the gate operations are likely to have an advantage that is insensitive to stochastic operation errors $[15,16]$. A kind of adiabatic GQC based on the conditional Berry phase was first proposed $[6,8,10,11]$, while the adiabatic condition may not be satisfied in many realistic cases since it requires one to operate a quantum gate very slowly so that the relevant instantaneous energy eigenstates follow its Hamiltonian to evolve. On the one hand, faster operation leads to severe distortions in the expected outcome, while on the other hand, the operation must be completed within the decoherence time of the system. In order to overcome this disadvantage, another kind of quantum gates based on the nonadiabatic geometric phase was suggested $[7,9]$. These gates likely possess the virtues of both a fast running speed and intrinsic geometric features of the adiabatic GQC. It is remarked that a key point in the above conventional GQC schemes is to avoid or remove the dynamic phases $[6-11,17]$, so that only the geometric phases

*Electronic address: zwang@hkucc.hku.hk are accumulated in the whole gate operation. Apart from these methods, an unconventional GQC was also proposed to construct quantum gates in several physical systems $[18,19]$.

In this paper, we propose a strategy to implement a set of quantum gates based on the geometric phases accumulated in the nondegenerate eigenstates of a periodic invariant operator in a physical system. Let the system evolve in such an intriguing way that the dynamical phase shifts of the invariant operator eigenstates are identical (or $\bmod 2 \pi)$ while the corresponding geometric phases are nontrivial. In particular, we illustrate how to realize our scheme in a simple but typical NMR-type system. Certainly, the present strategy can also be applied to other systems.

Consider a physical system whose Hamiltonian $\hat{H}(t)$ and an invariant operator $\hat{I}(t)$ are time dependent and evolve periodically with periodicity $\tau[20]-$ i.e.,

$$
\hat{H}(0)=\hat{H}(\tau), \quad \hat{I}(0)=\hat{I}(\tau),
$$

where the invariant operator $\hat{I}$ is determined by $(\hbar=1)$

$$
\frac{\partial \hat{I}}{\partial t}-i[\hat{I}, \hat{H}] \equiv 0 .
$$

To realize geometric quantum gates based on the invariant operator strategy, we here focus only on the simple cases in which all eigenstates of $\hat{I}$ are nondegenerate. From Eq. (2) and the eigenvalue equation $\hat{I}|n, t\rangle_{I}=\lambda_{n}|n, t\rangle_{I}(n=1,2, \ldots)$, it is straightforward to summarize the following three properties of $\hat{I}$ [20-22]. (i) All eigenvalues $\left\{\lambda_{n}\right\}$ are time independent. (ii) The evolving state $|n, t\rangle_{S}=U(t, 0)|n, 0\rangle_{I}$ is always the eigenstate of $\hat{I}(t)$ with the same eigenvalue $\lambda_{n}$, where $U(t, 0)$ is the evolution operator satisfying the Schrödinger equation $i \frac{\partial}{\partial t} U=\hat{H} U$. Since both $|n, t\rangle_{S}$ and $|n, t\rangle_{I}$ are eigenstates of $\hat{I}(t)$ specified by the same eigenvalue $\lambda_{n}$, there exists a time-dependent gauge transformation between them:

$$
|n, t\rangle_{S}=e^{i \gamma_{n}(t)}|n, t\rangle_{I},
$$

with 


$$
\gamma_{n}(t)=\int_{0}^{t} d t^{\prime}\left\langle n, t^{\prime}\left|i \frac{\partial}{\partial t^{\prime}}-\hat{H}\left(t^{\prime}\right)\right| n, t^{\prime}\right\rangle_{I},
$$

where the phase $\left\{\gamma_{n}\right\}$ is referred to as the Lewis phase [21]. (iii) Transitions between the eigenstates specified by different eigenvalues are impossible, simply because the evolution operator represented in the basis $|n, t\rangle_{I}$ reads

$$
U_{I}(t, 0)=\left(\begin{array}{lll}
e^{i \gamma_{1}(t)} & & \\
& e^{i \gamma_{2}(t)} & \\
& & \ddots
\end{array}\right)_{I} .
$$

In addition, from the periodic condition given by Eq. (1), it is straightforward to derive a key relation $|n, \tau\rangle_{I}=|n, 0\rangle_{I}$, which plays an essential role in the present scheme.

Recently, a theory of geometric phases for invariant operators was developed [20]. The corresponding geometric phase is interpreted as a holonomy inherited from the universal Stiefel bundle over a Grassmann manifold. For a cyclic evolution of the eigenstate of $I(t)$, the Lewis phase in Eq. (4) is nothing but the total phase shift consisting of the geometric phase $\int_{0}^{\tau} d t^{\prime}\left\langle n, t^{\prime}\left|i \frac{\partial}{\partial t^{\prime}}\right| n, t^{\prime}\right\rangle I_{I}$ and the dynamic one $-\int_{0}^{\tau} d t^{\prime}\left\langle n, t^{\prime}\left|\hat{H}\left(t^{\prime}\right)\right| n, t^{\prime}\right\rangle_{I} \quad[14,20,22]$. To achieve quantum gates that depend only on geometric phases, we need to eliminate the above dynamic phases. We consider a system whose space bases are the normalized nondegenerate eigenstates of $\hat{I}(0)$. An arbitrary initial state in the system can be written as $|\Psi(0)\rangle=\Sigma_{n} c_{n}|n, 0\rangle_{I}$, where $\left\{c_{n}\right\}$ are the expansion coefficients. After operating a periodic evolution as given in Eq. (1), the final state becomes

$$
|\Psi(\tau)\rangle=\sum_{n} c_{n} e^{i \gamma_{n}(\tau)}|n, 0\rangle_{I},
$$

where $\gamma_{n}(\tau)$ is the total phase shift of the state $|n, 0\rangle_{I}$ which consists of the dynamic part $\gamma_{n}^{d}(\tau)$ and the geometric part $\gamma_{n}^{g}(\tau)[12,14]$. Here, we have used the condition $|n, \tau\rangle_{I}$ $=|n, 0\rangle_{I}$. If the accumulated dynamic phases of $\left\{|n, 0\rangle_{I}\right\}$ are identical (or $\bmod 2 \pi$ ), namely,

$$
\gamma_{n}^{d}(\tau)=\gamma_{0}+2 K_{n} \pi
$$

where $K_{n}$ is an integer, the final state $|\Psi(\tau)\rangle$ is given by

$$
|\Psi(\tau)\rangle=e^{i \gamma_{0}} \sum_{n} c_{n} e^{i \gamma_{n}^{g}(\tau)}|n, 0\rangle_{I} .
$$

Note that the overall identical phase shift $\gamma_{0}$ in Eq. (8) is irrelevant to the designed quantum computation, and thus only the geometric phase is relevant to the gate operation, which is just a key idea to construct, in principle, geometric quantum gates [23]. In fact, the present geometric strategy is to operate quantum gates in such a way that the nondegenerate eigenstates of the invariant operator accumulate the same dynamic phase but with nontrivial relative geometric phases.

As a simple but typical example, we now illustrate how to implement the above generic strategy in an NMR-type qubit system, noting that the NMR approach has been a mature technique to simply simulate and examine quantum information processing schemes. Certainly, the present scheme is also applicable in principle to other quantum systems that evolve nonadiabatically. Consider an NMR-type spin-1/2 system, subject to a rotating magnetic field given by

$$
\vec{B}(t)=\left(-B_{2} \cos \omega t,-B_{2} \sin \omega t,-B_{1}\right),
$$

where $B_{1}$ and $B_{2}$ are, respectively, the amplitudes of the $z$ and $x y$-plane components of the field. The corresponding qubit Hamiltonian can be written as

$$
\hat{H}(t)=-\frac{1}{2} \mu_{B} \vec{\sigma} \cdot \vec{B}=\frac{1}{2} \omega_{1} \sigma_{z}+\frac{1}{2} \omega_{2}\left(\begin{array}{cc}
0 & e^{-i \omega t} \\
e^{i \omega t} & 0
\end{array}\right),
$$

where $\sigma_{x, y, z}$ are Pauli matrices, and $\omega_{1}=\mu_{B} B_{1}$ and $\omega_{2}$ $=\mu_{B} B_{2}$ with $\mu_{B}$ as the Bohr magneton. The corresponding evolution operator is [24]

$$
U(t, 0)=e^{-i \omega t \sigma_{z} / 2} e^{-i H_{0} t},
$$

where $\hat{H}_{0}=\hat{H}(0)-\omega \sigma_{z} / 2$ is just the Hamiltonian denoted in the rotating framework. An invariant operator satisfying Eq. (2) is then found to be

$$
\hat{I}=\frac{1}{2}\left(\omega_{1}-\omega\right) \sigma_{z}+\frac{1}{2} \omega_{2}\left(\begin{array}{cc}
0 & e^{-i \omega t} \\
e^{i \omega t} & 0
\end{array}\right) .
$$

Obviously, $\hat{I}(0)=\hat{H}_{0}$, and the invariant operator follows the Hamiltonian to evolve cyclically with periodicity $\tau=2 \pi / \omega$. The eigenvalues of $\hat{I}$ are evaluated to be $\pm \lambda / 2$ with $\lambda=\sqrt{\omega_{2}^{2}+\left(\omega_{1}-\omega\right)^{2}}$, and the two corresponding eigenstates are derived as $\left|\frac{1}{2} \lambda, t\right\rangle_{I}=\cos \frac{\chi}{2}|\uparrow\rangle+e^{i \omega t} \sin \frac{\chi}{2}|\downarrow\rangle$ and $\left|-\frac{1}{2} \lambda, t\right\rangle_{I}$ $=-\sin \frac{\chi}{2}|\uparrow\rangle+e^{i \omega t} \cos \frac{\chi}{2}|\downarrow\rangle$, where $\chi=2 \arctan \frac{\lambda+\omega-\omega_{1}}{\omega_{2}}$, and $|\uparrow\rangle$ and $|\downarrow\rangle$ are the two eigenstates of $\sigma_{z}$. Since $\hat{I}(0)=\hat{H}_{0}$, $\left| \pm \frac{1}{2} \lambda, 0\right\rangle_{I}$ are also the eigenstates of $H_{0}$. For a cyclic evolution and in the basis $\left| \pm \frac{1}{2} \lambda, 0\right\rangle_{I}$, the evolution operator $U_{I}(\tau)$ can be simply written as

$$
U_{I}(\tau, 0)=\left(\begin{array}{cc}
e^{i \pi(1-\lambda / \omega)} & 0 \\
0 & e^{i \pi(1+\lambda / \omega)}
\end{array}\right)_{I} .
$$

Note that, if we choose the computation basis as $|\uparrow\rangle$ and $|\downarrow\rangle$, the unitary transformation $\tilde{U}(\tau, 0)$ between the input and output states can also be given explicitly [25]:

$$
\tilde{U}=\left(\begin{array}{cc}
e^{i \gamma} \cos ^{2} \frac{\chi}{2}+e^{-i \gamma} \sin ^{2} \frac{\chi}{2} & i \sin \chi \sin \gamma \\
i \sin \chi \sin \gamma & e^{i \gamma} \sin ^{2} \frac{\chi}{2}+e^{-i \gamma} \cos ^{2} \frac{\chi}{2}
\end{array}\right),
$$

where $\gamma=\gamma_{+}=\pi(1-\lambda / \omega)$ is the total phase shift of $\left|\frac{1}{2} \lambda, 0\right\rangle_{I}$ in one cyclic evolution.

Similarly, the total phase shift of $\left|-\frac{1}{2} \lambda, 0\right\rangle_{I}$ is expressed as $\gamma_{-}=\pi(1+\lambda / \omega)$ in the cyclic evolution. The corresponding dynamic phases are derived to be

$$
\gamma_{ \pm}^{d}=\mp \pi \frac{\sqrt{\omega_{1}^{2}+\omega_{2}^{2}}}{\omega} \cos (\chi-\theta),
$$

with $\theta=\arctan \left(\omega_{2} / \omega_{1}\right)$. Here $(\chi-\theta)$ is just the angle between the magnet field and the state vector in the Bloch sphere as 
the eigenstate of $\hat{I}$ rotates with $\hat{H}$. The geometric phases of $\left| \pm \frac{1}{2} \lambda, 0\right\rangle_{I}$ are found to be

$$
\gamma_{ \pm}^{g}=\pi(1 \pm \cos \chi) .
$$

Using Eq. (7) to eliminate the dynamic phases in the gate operation, we are able to derive a relation for three parameters $\omega_{1}, \omega_{2}$, and $\omega$ :

$$
\frac{\left(\lambda+\omega-\omega_{1}\right)\left(\omega_{1}^{2}-\omega \omega_{1}+\omega_{2}^{2}\right)}{\omega_{2}^{2}+\left(\lambda+\omega-\omega_{1}\right)^{2}}=\frac{K \omega}{2},
$$

where $K$ is an integer. In the simplest case of $K=0$, we have $\omega_{1}^{2}+\omega_{2}^{2}=\omega \omega_{1}$. The geometric phases are simply given by

$$
\gamma_{ \pm}^{g}=\pi\left(1 \mp \frac{\lambda}{\omega}\right)=\pi(1 \pm \cos \chi) .
$$

Comparing with existing GQC schemes [9,25], the present strategy is simpler and more operable. Also, interestingly, in the adiabatic evolution-i.e., $\omega \ll \omega_{1}, \omega_{2}$-one has $\partial \hat{I} / \partial t \approx 0$, so that $[\hat{I}, \hat{H}] \approx 0$ and $\hat{I} \approx \hat{H}$ in the present example. In this case, the two eigenstates of $\hat{I}$ are also the instantaneous eigenstates of $\hat{H}$ and the conventional Berry phase is recovered [20]. Moreover, under the adiabatic approximation, a set of adiabatic Abelian geometric gates can be constructed more rigorously using the present theory plus a twoloop gate operation that can simply eliminate the dynamic phase.

At this stage, we elaborate how to realize a universal twoqubit quantum gate-namely, to construct a controlled- $U$ gate given by the following unitary transformation:

$$
U_{c}(T)=\left(\begin{array}{cc}
E & O \\
O & U
\end{array}\right),
$$

where $E$ and $O$ represent, respectively, the $2 \times 2$ unitary and zero matrices and $T$ is the operation periodicity of the gate. For simplicity but without loss of generality, we consider that the two spin-1/2 systems are coupled by an ordinary $-J \sigma_{1 z} \sigma_{2 z} / 2$ term with the coupling strength $J$. We also set $B_{1}=0$ for each single qubit and let the two resonant magnetic fields be applied only on the first qubit. The total Hamiltonian for this two-qubit system reads

$$
\hat{H}_{t}=-\frac{1}{2} J \sigma_{1 z} \sigma_{2 z}+\frac{1}{2} \omega_{0}\left(\sigma_{1 x} \cos \omega t+\sigma_{1 y} \sin \omega t\right),
$$

where $\sigma_{1}$ and $\sigma_{2}$ are the Pauli matrices for qubits 1 and 2,

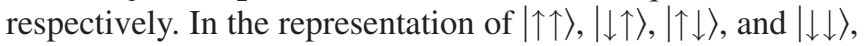
$\hat{H}_{t}$ can be decomposed into a direct product of the two single-qubit Hamiltonians as

$$
\hat{H}_{t}=\hat{H}_{1} \otimes \hat{H}_{2},
$$

where $\hat{H}_{1}$ and $\hat{H}_{2}$ are written as

$$
\hat{H}_{1}=\frac{1}{2}\left(\begin{array}{cc}
-J & \omega_{0} e^{-i \omega t} \\
\omega_{0} e^{i \omega t} & J
\end{array}\right)
$$

and

$$
\hat{H}_{2}=\frac{1}{2}\left(\begin{array}{cc}
J & \omega_{0} e^{-i \omega t} \\
\omega_{0} e^{i \omega t} & -J
\end{array}\right) .
$$

Clearly, $\hat{H}_{1}$ corresponds to the subspace spanned by bases $|\uparrow \uparrow\rangle$ and $|\downarrow \uparrow\rangle$, while $\hat{H}_{2}$ is in the subspace spanned by bases $|\uparrow \downarrow\rangle$ and $|\downarrow \downarrow\rangle$. These two subspaces are orthogonal. The invariant operator in Eq. (2) is found to be

$$
I_{t}=I_{1} \otimes I_{2}
$$

where

$$
I_{1}=\frac{1}{2}\left(\begin{array}{cc}
-J-\omega & \omega_{0} e^{-i \omega t} \\
\omega_{0} e^{i \omega t} & J+\omega
\end{array}\right)
$$

and

$$
I_{2}=\frac{1}{2}\left(\begin{array}{cc}
J-\omega & \omega_{0} e^{-i \omega t} \\
\omega_{0} e^{i \omega t} & -J+\omega
\end{array}\right) .
$$

The eigenvalues are $\pm \lambda_{1} / 2$ and $\pm \lambda_{2} / 2$, where $\lambda_{1}$ $=\sqrt{\omega_{0}^{2}+(J+\omega)^{2}}$ and $\lambda_{2}=\sqrt{\omega_{0}^{2}+(J-\omega)^{2}}$, respectively. The corresponding eigenstates of $I$ are $\left|\frac{1}{2} \lambda_{1}, t\right\rangle=\cos \frac{\chi_{1}}{2}|\uparrow \uparrow\rangle$ $+e^{i \omega t} \sin \frac{\chi_{1}}{2}|\uparrow \downarrow\rangle, \quad\left|-\frac{1}{2} \lambda_{1}, t\right\rangle=-\sin \frac{\chi_{1}}{2}|\uparrow \uparrow\rangle+e^{i \omega t} \cos \frac{\chi_{1}}{2}|\uparrow \downarrow\rangle$, $\left|\frac{1}{2} \lambda_{2}, t\right\rangle=\cos \frac{\chi_{2}}{2}|\uparrow \downarrow\rangle+e^{i \omega t} \sin \frac{\chi_{2}}{2}|\downarrow \downarrow\rangle \quad$ and $\quad\left|-\frac{1}{2} \lambda_{2}, t\right\rangle$ $=-\sin \frac{\chi_{2}}{2}|\uparrow \downarrow\rangle+e^{i \omega t} \cos \frac{\chi_{2}}{2}|\downarrow \downarrow\rangle$, where $\chi_{1}=2 \arctan \frac{\lambda_{1}+\omega+J}{\omega_{0}}$ and $\chi_{2}=2 \arctan \frac{\lambda_{2}+\omega-J}{\omega_{0}}$. For a cyclic evolution, the evolution operator of $\hat{H}_{t}$ in the representation of $\left| \pm \frac{1}{2} \lambda_{1}, 0\right\rangle$ and $\left| \pm \frac{1}{2} \lambda_{2}, 0\right\rangle$ can be written as

$$
U_{I}(\tau)=U_{1}(\tau) \otimes U_{2}(\tau),
$$

where

$$
U_{1}(\tau)=\left(\begin{array}{cc}
e^{i \pi\left(1-\lambda_{1} / \omega\right)} & 0 \\
0 & e^{i \pi\left(1+\lambda_{1} / \omega\right)}
\end{array}\right)_{I}
$$

and

$$
U_{2}(\tau)=\left(\begin{array}{cc}
e^{i \pi\left(1-\lambda_{2} / \omega\right)} & 0 \\
0 & e^{i \pi\left(1+\lambda_{2} / \omega\right)}
\end{array}\right)_{I}
$$

By a close inspection of Eq. (22) and considering that $\lambda_{1}$ $\neq \lambda_{2}$, we find that once a multicycle evolution $T=m \tau$ is operated, one may be able to achieve a two-qubit gate given by Eq. (18). For such an operation, the unitary transformation is given by

$$
U_{I}(T)=U_{I}(m \tau)=U_{I}(\tau)^{m} .
$$

Theoretically, for a rational $\left(\lambda_{1} / \omega\right)$, one may assert the relation

$$
m \pi\left(1+\lambda_{1} / \omega\right)=2 N \pi
$$

to be satisfied, where $N$ is an integer. This condition makes $U_{1}$ a unitary matrix - namely,

$$
U_{I}(T)=\left(\begin{array}{cc}
E & O \\
O & U_{2}(m \tau)
\end{array}\right)_{I} .
$$

Correspondingly, in the bases $(|\uparrow \uparrow\rangle,|\downarrow \uparrow\rangle,|\uparrow \downarrow\rangle,|\downarrow \downarrow\rangle)$, we have also a controlled- $U$ gate in the form 


$$
\tilde{U}_{c}(T)=\left(\begin{array}{cc}
E & O \\
O & \tilde{U}_{2}(m \tau)
\end{array}\right)
$$

Under this multicycle operation, the dynamic phase of $U_{2}$ is $\gamma_{ \pm}^{d}(m \tau)=m \gamma_{ \pm}^{d}(\tau)$. Thus we choose the appropriate parameters to ensure Eq. (16)-i.e.,

$$
\frac{\left(\lambda_{2}+\omega-J\right)\left(J^{2}-J \omega+\omega_{0}^{2}\right)}{\omega_{2}^{2}+\left(\lambda_{2}+\omega-J\right)^{2}}=\frac{K \omega}{2 m} .
$$

When qubit 2 is down, the geometric phase for $K=0$ is

$$
\gamma_{2 \pm}^{g}=m \pi\left(1 \mp \frac{\lambda_{2}}{\omega}\right) \text {. }
$$

As a result, a universal geometric quantum gate is realized. For instance, if we set $K=0, N=4, J=\frac{16}{27} \omega$, and $\omega_{0}=\frac{4 \sqrt{11}}{27} \omega$, we could have $m=3$. The geometric phases are thus $\gamma_{2 \pm}^{g}$ $=\pi\left(1 \mp \sqrt{\frac{11}{3}}\right)$.
In summary, we have proposed a strategy to implement a set of quantum gates based on the geometric phases accumulated in the nondegenerate eigenstates of an invariant operator in a periodic physical system. An intriguing way is presented to eliminate the dynamical phase shifts in the designated gate operation. In addition, we have also illustrated how to implement our scheme in a simple but typical NMR-type system, while the present strategy may also be feasible in other systems.

\section{ACKNOWLEDGMENTS}

We thank J. C. Y. Teo for helpful discussions. This work was supported by the National Natural Science Foundation of China (Grant No. 10429401), the State Key Program for Basic Research of China (Grant No. 2006CB0L1001), the RGC of Hong Kong (Grant No. HKU 7045/05), and the URC fund of Hong Kong.
[1] R. Jozsa, Proc. R. Soc. London, Ser. A 439, 553 (1992).

[2] M. A. Nielsen and I. L. Chuang, Quantum Computation and Quantum Information (Cambridge University Press, Cambridge, England, 2000).

[3] J. I. Cirac and P. Zoller, Phys. Rev. Lett. 74, 4091 (1995).

[4] D. V. Averin, Solid State Commun. 105, 659 (1998).

[5] Y. Makhlin, G. Schön, and A. Shnirman, Nature (London) 398, 305 (1999).

[6] J. A. Jones, V. Vedral, A. Ekert, and G. Castagnoli, Nature (London) 403, 342 (2000).

[7] Xiang-Bin Wang and M. Keiji, Phys. Rev. Lett. 87, 097901 (2001).

[8] G. Falci, R. Fazio, G. M. Palma, J. Siewert, and V. Vedral, Nature (London) 407, 355 (2000).

[9] S. L. Zhu and Z. D. Wang, Phys. Rev. Lett. 89, 097902 (2002).

[10] L. M. Duan, J. I. Cirac, and P. Zoller, Science 292, 1695 (2001).

[11] P. Solinas, P. Zanardi, N. Zanghi, and F. Rossi, Phys. Rev. B 67, 121307(R) (2003).

[12] M. V. Berry, Proc. R. Soc. London, Ser. A 392, 45 (1984).

[13] B. Simon, Phys. Rev. Lett. 51, 2167 (1983).

[14] Y. Aharonov and J. Anandan, Phys. Rev. Lett. 58, 1593 (1987).

[15] P. Solinas, P. Zanardi, and N. Zanghi, Phys. Rev. A 70, 042316
(2004).

[16] S. L. Zhu and P. Zanardi, Phys. Rev. A 72, 020301(R) (2006).

[17] S. L. Zhu and Z. D. Wang, Phys. Rev. A 67, 022319 (2003).

[18] S. L. Zhu and Z. D. Wang, Phys. Rev. Lett. 91, 187902 (2003); S. L. Zhu, Z. D. Wang, and P. Zanardi, ibid. 94, 100502 (2005).

[19] D. Leibfried, B. Demarco, V. Meyer, D. Lucas, M. Barrett, J. Britton, W. M. Itano, B. Jelenkovic, C. Langer, T. Rosenband, and D. J. Wineland, Nature (London) 422, 412 (2003).

[20] J. C. Y. Teo and Z. D. Wang, Phys. Rev. Lett. 95, 050406 (2005).

[21] H. R. Lewis and W. B. Riesenfeld, J. Math. Phys. 10, 1458 (1969).

[22] X. C. Gao, J. B. Xu, and T. Z. Qian, Phys. Rev. A 44, 7016 (1991).

[23] Since the complex terms with the phase difference $2 K \pi$ are actually equivalent both mathematically and physically, the integer $K$ has no real physical implication in the (dynamic) phase space, and thus it is believed that stochastic errors are unlikely to be manifested in the integer- $K$ cases, as for the case of $K$ $=0$.

[24] L. M. K. Vandersypen and I. L. Chuang, Rev. Mod. Phys. 76, 1037 (2004).

[25] X. D. Zhang, S. L. Zhu, L. Hu, and Z. D. Wang, Phys. Rev. A 71, 014302 (2005). 